\title{
Communication
}

\section{Transcriptomic Hallmarks of Ischemia-Reperfusion Injury}

\author{
Mandana Movahed ${ }^{1,2,+}$, Sydney Brockie ${ }^{1,2,+}$, James Hong ${ }^{1}$ and Michael G. Fehlings ${ }^{1,2,3, *}$ (i) \\ 1 Division of Genetics and Development, Krembil Research Institute, University Health Network, \\ Toronto, ON M5T 2S8, Canada; mandana.movahed@mail.utoronto.ca (M.M.); \\ s.brockie@mail.utoronto.ca (S.B.); jms.hong@mail.utoronto.ca (J.H.) \\ 2 Institute of Medical Science, University of Toronto, Toronto, ON M5S 1A8, Canada \\ 3 Spinal Program, University Health Network, Toronto Western Hospital, Toronto, ON M5T 2S8, Canada \\ * Correspondence: michael.fehlings@uhn.ca; Tel.: +1-416-603-5627 \\ + These authors contributed equally to this work.
}

check for updates

Citation: Movahed, M.; Brockie, S.; Hong, J.; Fehlings, M.G.

Transcriptomic Hallmarks of

Ischemia-Reperfusion Injury. Cells 2021, 10, 1838. https://doi.org/ 10.3390/cells10071838

Academic Editors: Antonio

Rodríguez-Sinovas,

Marisol Ruiz-Meana and

Javier Inserte

Received: 16 June 2021

Accepted: 9 July 2021

Published: 20 July 2021

Publisher's Note: MDPI stays neutral with regard to jurisdictional claims in published maps and institutional affiliations.

Copyright: (c) 2021 by the authors. Licensee MDPI, Basel, Switzerland. This article is an open access article distributed under the terms and conditions of the Creative Commons Attribution (CC BY) license (https:/ / creativecommons.org/licenses/by/ $4.0 /)$.

\begin{abstract}
Ischemia reperfusion injury (IRI) is associated with a broad array of life-threatening medical conditions including myocardial infarct, cerebral stroke, and organ transplant. Although the pathobiology and clinical manifestations of IRI are well reviewed by previous publications, IRI-related transcriptomic alterations are less studied. This study aimed to reveal a transcriptomic hallmark for IRI by using the RNA-sequencing data provided by several studies on non-human preclinical experimental models. In this regard, we focused on the transcriptional responses of IRI in an acute time-point up to $48 \mathrm{~h}$. We compiled a list of highly reported genes in the current literature that are affected in the context of IRI. We conducted Gene Ontology (GO) and Kyoto Encyclopedia of Genes and Genomes (KEGG) enrichment analyses and found many of the up-regulated genes to be involved in cell survival, cell surface signaling, response to oxidative stress, and inflammatory response, while down-regulated genes were predominantly involved in ion transport. Furthermore, by GO analysis, we found that multiple inflammatory and stress response processes were affected after IRI. Tumor necrosis factor alpha (TNF) and nuclear factor kappa-light-chain-enhancer of activated B cells (NF- $\mathrm{kB}$ ) signaling pathways were also highlighted in the Kyoto Encyclopedia of Genes and Genomes enrichment analysis. In the last section, we discuss the treatment approaches and their efficacy for IRI by comparing RNA sequencing data from therapeutic interventions with the results of our cross-comparison of differentially expressed genes and pathways across IRI.
\end{abstract}

Keywords: ischemia reperfusion injury; RNA-seq; transcriptomics

\section{Introduction}

Ischemia reperfusion injury (IRI) is imposed by the transient loss of circulation to a specific tissue for various durations of time, followed by the restoration of blood flow that results in widespread inflammatory responses causing secondary injury [1]. IRI commonly occurs in myocardial infarction [2], cerebral stroke [3], and cases of transplantation of the kidney [4] and liver [5]. Across these injury modalities, loss of circulation causes ischemic hypoxia, which has vast effects in cell metabolism, ionic balance maintenance, and mitochondrial function [1].

In hypoxic conditions, glycolysis becomes the sole energy-producing process and the mitochondrial electron transport system is inhibited, resulting in buildup of glycolytic by-products and reactive oxidative species (ROS) [6]. This disrupts ionic balances, thereby disrupting transmembrane potential. In the mitochondria, a lack of oxygen causing buildup of hydrogen ions across the membrane may cause the mitochondrial permeability transition pore to open, decreasing transmembrane potential and ultimately resulting in bursting and subsequent release of apoptogens and organelle components, contributing to damageassociated molecular patterns [6]. Similarly, buildup of ROS caused by mitochondrial dysfunction impairs protein folding in the endoplasmic reticulum [7] and activate protein 
complexes, such as mitogen-activated protein kinase (MAPK) [8], calcium/calmodulindependent protein kinase (CaMK) [9], protein kinase C [10], and receptor-interacting protein kinases [11], which initiate several pro-inflammatory cascades. Ionic imbalances inflict similar damage across the cell membrane, where voltage-gated channels respond to the absence of oxygen by increasing ionic exchange to maintain tonicity, eventually leading to excess intracellular calcium and cytotoxicity [12]. In cases of stroke, the dramatic release of glutamate from calcium-saturated cells causes widespread neuronal glutamatergic excitotoxicity and cell death, and inhibiting sodium channels with was found to attenuate this cascade [13].

Ischemia and ischemia reperfusion effects were also observed on the genomic level, where ROS were found to drive epigenetic changes in expression of mRNA [14] and noncoding RNAs, facilitating an array of further cellular responses [1]. While the molecular footprint of IRI was extensively studied and articulated, transcriptional responses have only recently garnered attention and lack any in-depth characterization. Moreover, these transcriptional events are likely to play a far more significant role than is currently appreciated, and understanding them is critical to addressing the pathophysiology of IRI [14]. Here, we will review several recent studies in order to reveal a transcriptomic hallmark for IRI during acute time-point. We will do this by comparing the differential expression of genes reported after the onset of IRI. Table 1 presents a summary of the articles that are used in the paper.

Table 1. Summary of studied articles in this study.

\begin{tabular}{|c|c|c|c|}
\hline References & Reperfusion Model & Species & Reperfusion Duration \\
\hline Zhou et al. 2020 [15] * & Spinal cord IRI & Sprague-Dawley rat & $48 \mathrm{~h}$ \\
\hline Cai et al. 2019 [16] * & Cerebral IRI & C57BL/6J mice & $1,3,7,14,28 \mathrm{~d}$ \\
\hline Dergunova et al. 2018 [17] * & Cerebral IRI & Wistar rats & $4.5,24 \mathrm{~h}$ \\
\hline Zhang et al. 2019 [18]* & Hepatic IRI & C57BL/6J mice & $4 \mathrm{~h}$ \\
\hline Shi et al. 2017 [19] * & Cerebral IRI & Sprague-Dawley rat & $45 \mathrm{~min}, 6 \mathrm{~h}, 12 \mathrm{~h}$ and $18 \mathrm{~h}$ \\
\hline Johnsen et al. 2020 [20] * & Renal IRI & C57BL/6J mice & $4,24 \mathrm{~h}$ \\
\hline Li et al. 2020 [21] * & Myocardial IRI & C57BL/6J mice & $40 \mathrm{~min}$ \\
\hline Mo et al. 2019 [22] & Myocardial IRI & C57BL/6J mice & $1 \mathrm{~h}$ \\
\hline Kestner et al. 2020 [23] & Cerebral IRI & C57BL/6J mice & $24 \mathrm{~h}$ \\
\hline Kolling et al. 2018 [24] & Renal IRI & C57BL/6J mice & $24 \mathrm{~h}$ \\
\hline Wang et al. 2017 [25] & Cerebral IRI & Wistar rats & $24 \mathrm{~h}$ \\
\hline Park et al. 2020 [26] & Renal IRI & Human & $10 \mathrm{~min}$ \\
\hline Giraud et al. 2018 [27] & Renal IRI & Porcine & $6,24 \mathrm{~h}$ \\
\hline Deng et al. 2020 [28] & Myocardial IRI & C57BL/6J mice & $24 \mathrm{~h}$ \\
\hline Smith et al. 2018 [29] & Cerebral IRI & C57BL/6J mice & $24 \mathrm{~h}, 48 \mathrm{~h}, 1 \mathrm{w}, 2 \mathrm{w}$ \\
\hline Sun et al. 2018 [30] & Hepatic IRI & Sprague-Dawley rats & $6,24,48 \mathrm{~h}$ \\
\hline Liu et al. 2017 [31] & Renal IRI & C57BL/6J mice & $\begin{array}{c}2 \mathrm{~h}, 4 \mathrm{~h}, 1 \mathrm{~d}, 2 \mathrm{~d}, 3 \mathrm{~d}, 1 \mathrm{w}, 2 \mathrm{w}, \\
4 \mathrm{w}, 6 \text { months, } 12 \text { months }\end{array}$ \\
\hline
\end{tabular}

${ }^{*}$ The RNA-seq data from these articles were used in the comparison analysis.

\section{Methods}

\subsection{Systematic Review}

The PubMed database (https:/ / pubmed.ncbi.nlm.nih.gov/, accessed on 14 April 2021) was thoroughly searched from inception to April 2021. Three searches were conducted using the following key words in either the abstract or the title: ("IRI" AND "RNAsequencing"), ("ischemia reperfusion injury" AND "RNA-sequencing"), and ("ischemia reperfusion injury" AND "transcriptomic"). The selected articles had to contain open access datasets with the full list of differentially expressed genes (DEGs) from bulk RNAsequencing. Studies on single-cell RNA-sequencing were excluded. The changes in the mRNA profile were extracted for the acute phase (from the injury onset up to $48 \mathrm{~h}$ ) post-IRI. Selected articles also had to contain either naïve control or uninjured sham in order to compare the normal gene expression profile and the IRI-related changes in the mRNA 
profile. The studies reporting gene expression changes only after applying a treatment for IRI were excluded.

\subsection{Data Extraction and Handling}

We extracted the DEGs (adjusted $p$-value $<0.05$ ) from each publication and crossreferenced them based on the reported gene symbols. The resultant list included genes reported with varying frequencies (scores) across studies ranging from 7 to 1 . Next, this list was filtered for consistency. We kept only the genes that had consistent expression directionality in at least $75 \%$ of the reports for that gene. We then divided these genes into up- and down-regulated lists. Finally, we ranked these genes based on the number of times they were reported across studies. The genes with 4 or more reports (out of 7) were identified as "commonly reported DEGs" (Tables S4 and S5), which were used for further enrichment analysis. The highest ranked items of up- and down-regulated lists were represented as "highly reported DEGs" in Tables 2 and 3. All the initial data handling was performed on Excel version 2016 (Microsoft; WA, USA).

Table 2. Highly repeated up-regulated genes in IRI.

\begin{tabular}{|c|c|}
\hline Upregulated & Comments \\
\hline Itga5 & Programmed cell death \\
\hline Atf3 & Cell proliferation, programmed cell death \\
\hline Cyr61 & Cell proliferation, programmed cell death \\
\hline Litaf & Programmed cell death \\
\hline Stat3 & Programmed cell death, inflammatory response \\
\hline Icam1 & Inflammatory response, programmed cell death \\
\hline Sik1 & Programmed cell death \\
\hline $\operatorname{Tl} 2$ & Inflammatory response \\
\hline Zc3h12a & Inflammatory response \\
\hline Epha2 & Inflammatory response \\
\hline Maff & Response to stress \\
\hline Nes & Cell proliferation, programmed cell death \\
\hline Hmox1 & Response to stress, inflammatory response \\
\hline Myc & Programmed cell death, response to stress \\
\hline Cd44 & Inflammatory response \\
\hline Hbegf & Cell proliferation \\
\hline Ifrd1 & Developmental process \\
\hline$P d p n$ & Cell proliferation, programmed cell death \\
\hline Cd14 & Inflammatory response \\
\hline Jun & Cell proliferation, programmed cell death \\
\hline Csf1 & Inflammatory response \\
\hline Gadd $45 a$ & Programmed cell death, response to stress \\
\hline Ccl2 & Inflammatory response \\
\hline Klf6 & Developmental process \\
\hline Hspb1 & Response to stress, programmed cell death \\
\hline Olr1 & Inflammatory response \\
\hline Mafk & Response to stress \\
\hline$Z y x$ & Inflammatory response \\
\hline Osmr & Inflammatory response \\
\hline Tifa & Response to stress \\
\hline Bcl3 & Inflammatory response, programmed cell death \\
\hline Irf1 & Cell proliferation, programmed cell death \\
\hline Tnfrsfla & Inflammatory response \\
\hline Cxcl1 & Inflammatory response \\
\hline Txnrd1 & Cell proliferation, response to stress \\
\hline Spon2 & Cell surface adhesion and signaling, response to stress \\
\hline Myd88 & Inflammatory response \\
\hline Bach1 & Response to stress \\
\hline
\end{tabular}


Table 3. Highly repeated down-regulated genes in IRI.

\begin{tabular}{cc}
\hline Downregulated & Comments \\
\hline Cabp1 & Voltage-gated calcium ion channel regulator \\
Pclo & Presynaptic cytoskeletal matrix \\
Npy1r & Synaptic signaling \\
Tenm2 & Formation of growth cone in neural cells \\
Cbx7 & Cellular lifespan regulator \\
Camk4 & Transcriptional regulation \\
Kcng1 & Voltage-gated potassium (Kv) channels subunit \\
L1cam & Cell surface adhesion and signaling \\
Dlg2 & Response to oxidative stress and DNA damage \\
Scn4b & Sodium channel beta subunit \\
Kcnab1 & Cytoplasmic potassium channel subunit \\
Rasgrp1 & T cell/B cell regulator \\
Kcnt1 & Potassium-sodium activated channel subunit \\
Adora2a $a$ Cell surface adhesion and signaling & Endothelial cell survival \\
Phactr1 & Maturation of dendritic spines \\
Lts3 & Cell surface adhesion and signaling \\
Agrn & Cytoplasmic Ca ion regulator \\
Cacnb3 & Coltage-dependent calcium channel regulatory subunit \\
Cobl & Reorganization of the actin cytoskeleton \\
\hline
\end{tabular}

\subsection{GGO Enrichment Analysis}

The GO categories "Molecular Function (MF)", "Biological Process (BP)", and "Cellular Components (CC)" were analyzed for significantly enriched terms using the PANTHER enrichment test [32]. The analysis was run against the Mus musculus reference list using Fisher's exact test and p-values were corrected by false discovery rate (FDR) calculation. Finally, all terms with an FDR $<0.05$ were collected and the top 20 terms for each category were graphed using the ggplot2 package in $\mathrm{R}$ [33].

\subsection{KEGG Analysis}

The most highly affected KEGG pathways were isolated using the clusterProfiler package in R [33,34]. Commonly repeated DEGs were enriched with KEGG dataset for the Mus musculus organism (organism code: $\mathrm{mmu}$ ) and pathways with an adjusted $p$-value of $<0.05$ were plotted. Finally, the top 20 significant KEGG pathways were graphed on bubble charts using the ggplot2 package in R.

\subsection{Venn Diagram}

We graphed the DEG lists from all studied publications (genes with adjusted $p$-values of $<0.05$ ) using the Venn diagrams drawer online tool (http:/ / bioinformatics.psb.ugent. be/webtools/Venn/, accessed on 20 June 2021). To analyze organ-specific changes, we combined the DEG lists from studies on cerebral models (three publications) and used this in our comparison across organ models (spinal cord, hepatic, renal, and myocardial; one publication per organ).

\section{Results}

\subsection{Transcriptomic Hallmark of Ischemic Reperfusion Injury}

The injurious effects of reperfusion injury appear over hours and days following ischemia. In this article, we first aimed to outline the transcriptomic hallmark of IRI occurring over the 48-h window post-ischemia. We did this by conducting a comparison analysis between the DEGs of mRNA sequencing data provided by multiple studies on non-human preclinical experimental models (full list is provided in Table S1). In our comparative analysis, we only considered the genes that were consistently reported to be either up- or down-regulated. The genes with the highest consistency score are presented as "highly repeated DEGs" in Tables 2 and 3. Among the upregulated genes, there are multiple 
inflammatory response genes, including Bcl3, Tnfrsf1a, Cxcl1, and Tlr2. Furthermore, the upregulation of Litaf, Myc, Stat3, Atf3, and Cyr61 suggested the involvement of cell survival and apoptotic pathways. Other contributing processes may be cell surface adhesion and signaling (Icam1, Spon2, and Itga5) and oxidative stress response (Hspb1 and Hmox1). Interestingly, most of the consistently downregulated genes related to ion channels (e.g., $S c n 4 b, C a b p 1, K c n a b 1, K c n t 1, K c n g 1$, and Cacnb3). Other downregulated genes play roles in cytoskeleton regulation (Pclo and $\mathrm{Cobl}$ ) and response to hypoxia and DNA damage (Dlg2).

Although the selected articles for comparative analysis performed bulk RNA sequencing, we noticed several of our highly repeated DEGs matched with single-cell RNA sequencing results from Zamanian et al., 2012. In this study, the authors isolated astrocytes from an ischemic condition, and reported differential expression patterns [35]. The results suggested that astrocytes from the ischemic condition displayed a protective phenotype with a specific gene expression pattern from other types of injuries, such as neuroinflammation. Notably, some of our highly repeated DEGs were similar to the reported genes for these protective astrocytes from the ischemic condition (Litaf, Bcl3, Hmox1, Myc, Stat3, Atf3, Icam1, Tnfrsf1a, Cxcl1, and Hspb1). This information supports the notion that astrocytes may be one of the key contributors to IRI and likely play a protective role in this context.

\subsection{Pathways Involved in Ischemia Reperfusion Injury}

Next, we used a list of "commonly reported DEGs" in IRI conditions (4 out of 7) to run functional enrichment analysis. This list consisted of 571 upregulated and 135 downregulated genes (Tables S4 and S5). GO enrichment analysis was used to study biological processes, cellular components, and molecular functions implicated in IRI. The top 20 up- and down-regulated terms are reported in Figures 1 and 2, respectively. Among the most highly enriched biological processes, several upregulated DEGs related to immune signaling and cell death regulation, while downregulated DEGs were largely involved in intercellular signaling and synaptic transmission. The upregulated genes related to molecular functions primarily involved binding proteins, while downregulated genes related to transport channels. Finally, among enriched cellular component DEGs, those upregulated were found to relate to the cell periphery (GO:0071944), while those composing the synapse (GO:0045202) were downregulated.

Further, we studied the engaged pathways using KEGG enrichment analysis (Figure 3). The pathway analysis for upregulated genes highlighted multiple inflammatory response pathways, such as TNF, NF-kB, and IL-12 signaling pathways, as well as cytokine-cytokine receptor interaction, and toll-like receptor signaling. Conversely, the top dysregulated pathways involving significantly downregulated genes included oxytocin and calcium signaling pathways and long-term potentiation.

\subsection{Organ-Specific Differential Expression in Response to IRI}

To illustrate transcriptomic changes in each organ, we used a Venn diagram of DEGs from the selected studies (see Table 1) categorized by organ (details are provided in Table S2). We found 38 DEGs common across all five organs (Figure 4). Among these were Bcl3, Icam1, Itag5, Atf3, and Myc, which are involved in apoptosis and cell death. We also observed transcriptomic changes in genes involved in the inflammatory response, including Stat3, Tlr2, Cxcl1, Epha2, and Myd88. Furthermore, based on these 38 genes, TNF, NF- $\kappa B$, and MAPK signaling pathways appeared to be highly implicated in IRI response. 
A.

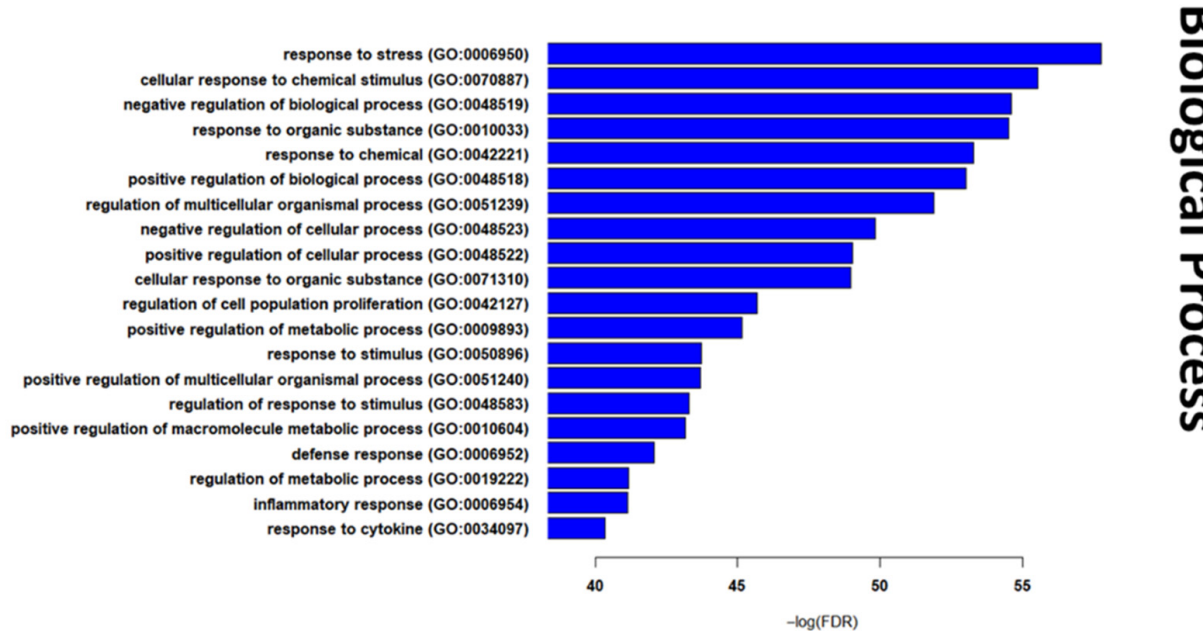

B.

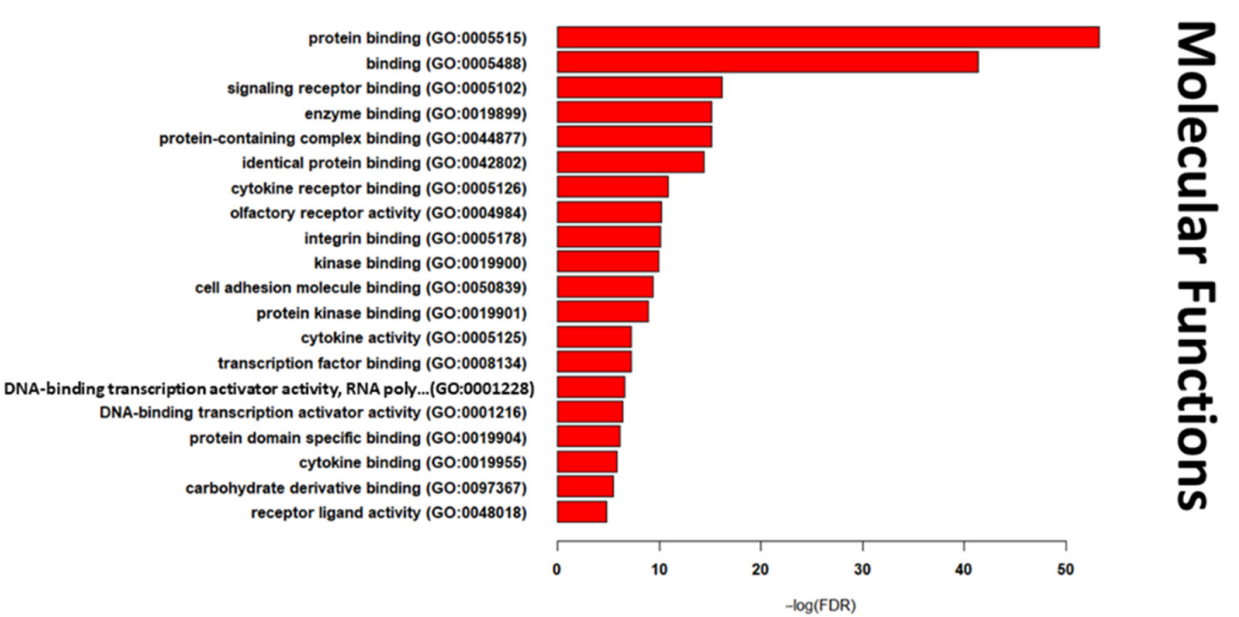

C.
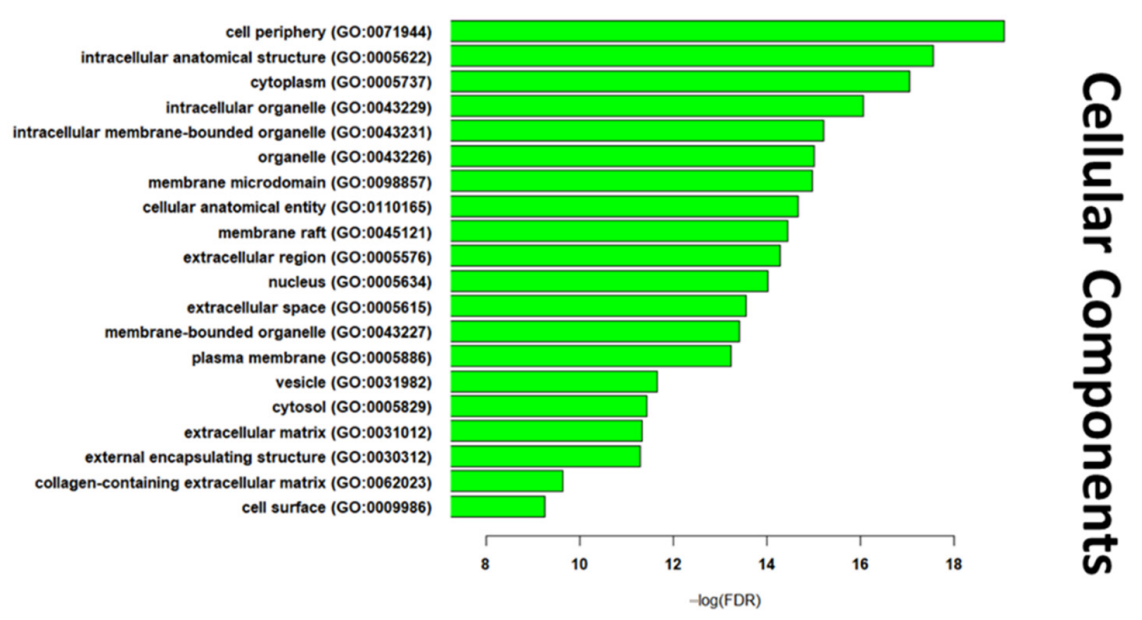

Figure 1. The top $20 \mathrm{GO}$ terms in each of the biological process (A), cellular components (B), and molecular functions (C) domains for upregulated genes (list of all significant terms can be found in Tables S6-S8). 
A.

B.

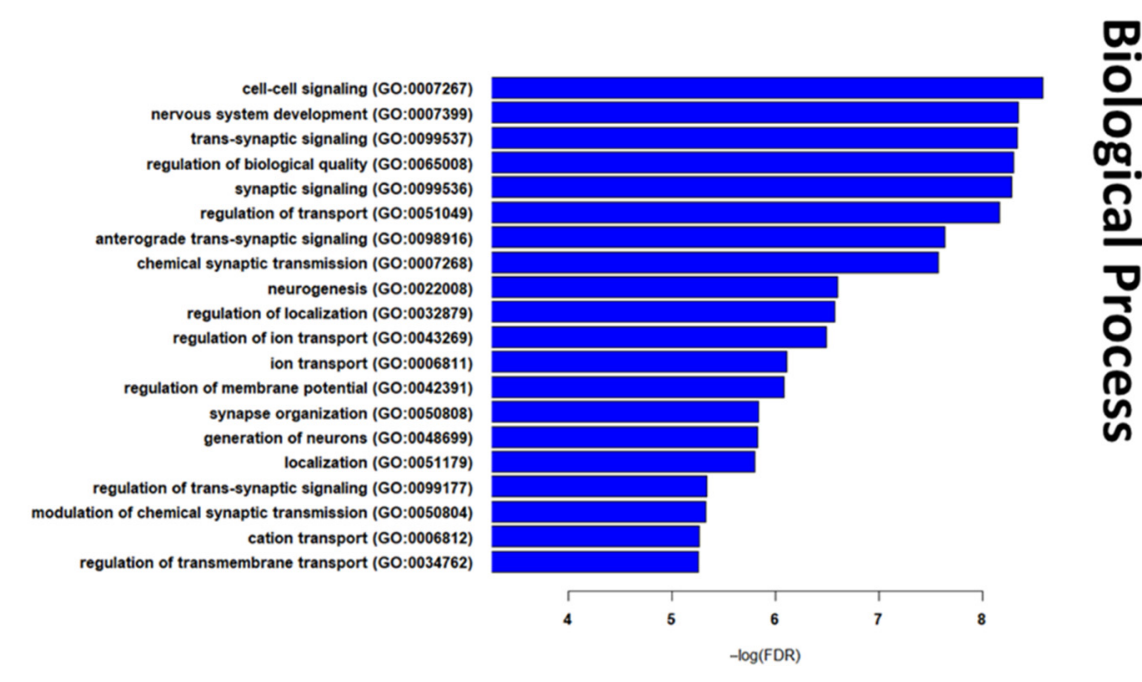
gated channel activity (GO:0022836) gated channel activity (GO:0022836)
cation channel activity (GO:0005261)
ion channel activity (GO:0005216)
metal ion transmembrane transporter activity (GO:0046873)
voltage-gated channel activity (GO:0022832)
voltage-gated ion channel activity (GO:0005244) gated channel activity (GO:0022836)
cation channel activity (GO:0005261)
ion channel activity (GO:0005216)
metal ion transmembrane transporter activity (GO:0046873)
voltage-gated channel activity (GO:0022832)
voltage-gated ion channel activity (GO:0005244) voltage-gated ion channel activity (GO:0005244) channel activity (GO:0015267) passive transmembrane transporter activity (GO:0022803) inorganic cation transmembrane transporter activity (GO:0022890) protein binding (GO:0005515)
voltage-gated cation channel activity (GO:0022843) ion transmembrane transporter activity (GO:0015075) cation transmembrane transporter activity (GO:0008324) inorganic molecular entity transmembrane transporter... (G0:0015318) ion channel regulator activity (GO:0099106) ion channetr transmembrane transporter activity (GO:0022857) potassium channel activity (GO:0005267) molecular function regulator (GO:0098772) transporter activity (GO:0005215) calcium ion binding (GO:0005509)

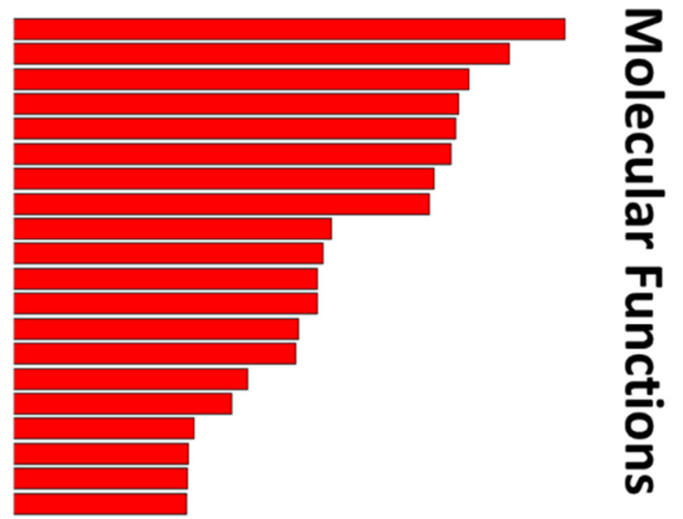

C.
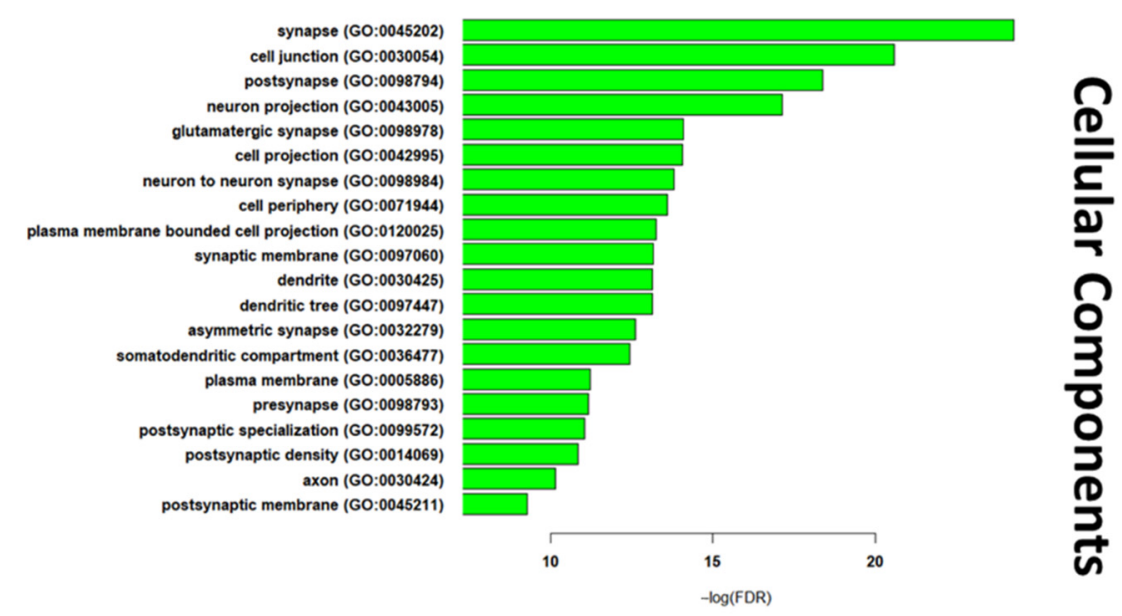

Figure 2. The top $20 \mathrm{GO}$ terms in each of the biological process (A), cellular components (B), and molecular functions (C) domains for downregulated genes (list of all significant terms can be found in Tables S9-S11). 


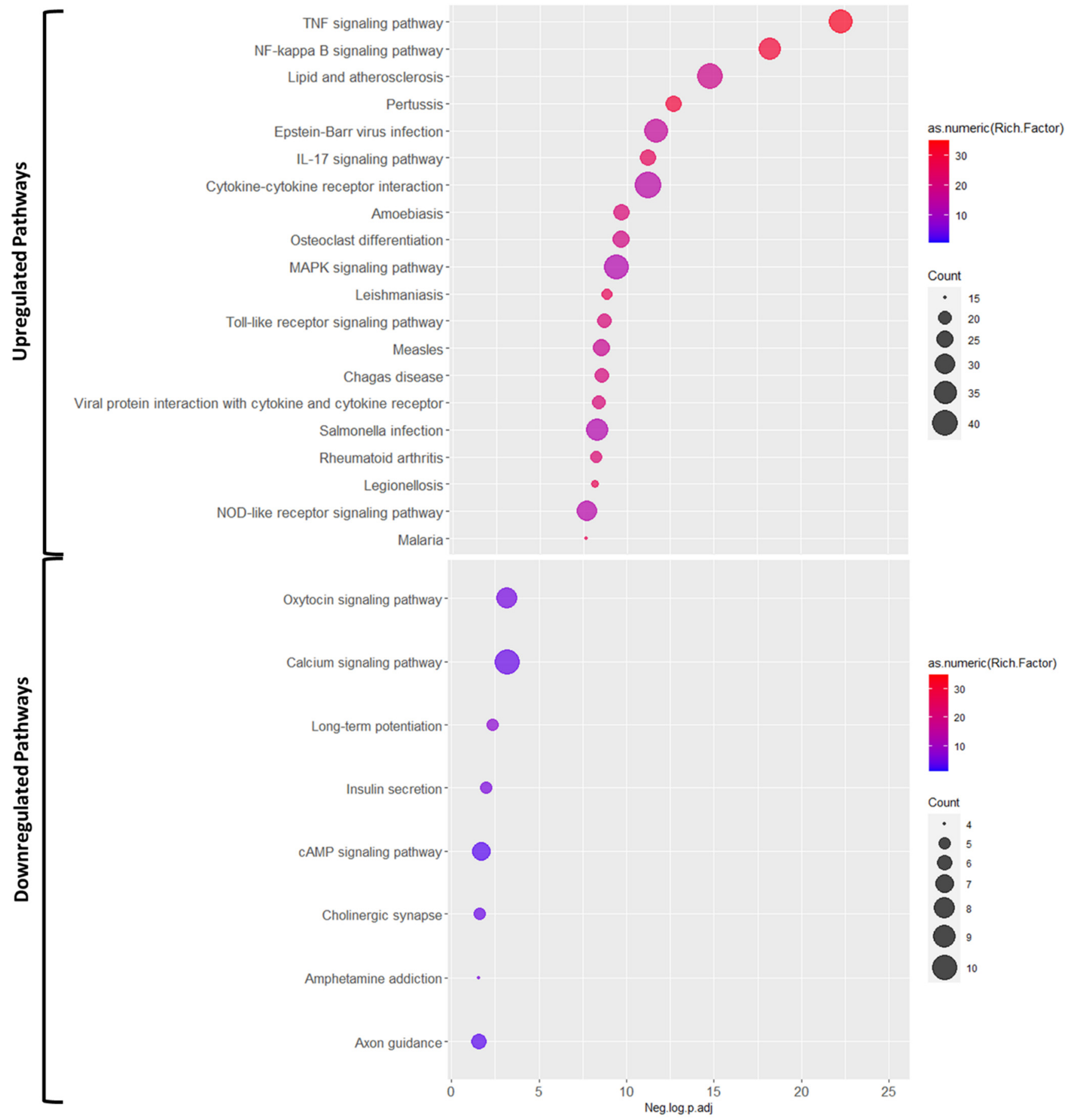

Figure 3. The results of KEGG enrichment pathway for upregulated and downregulated DEGs for IR condition (list of all significant terms can be found in Tables S12 and S13). 


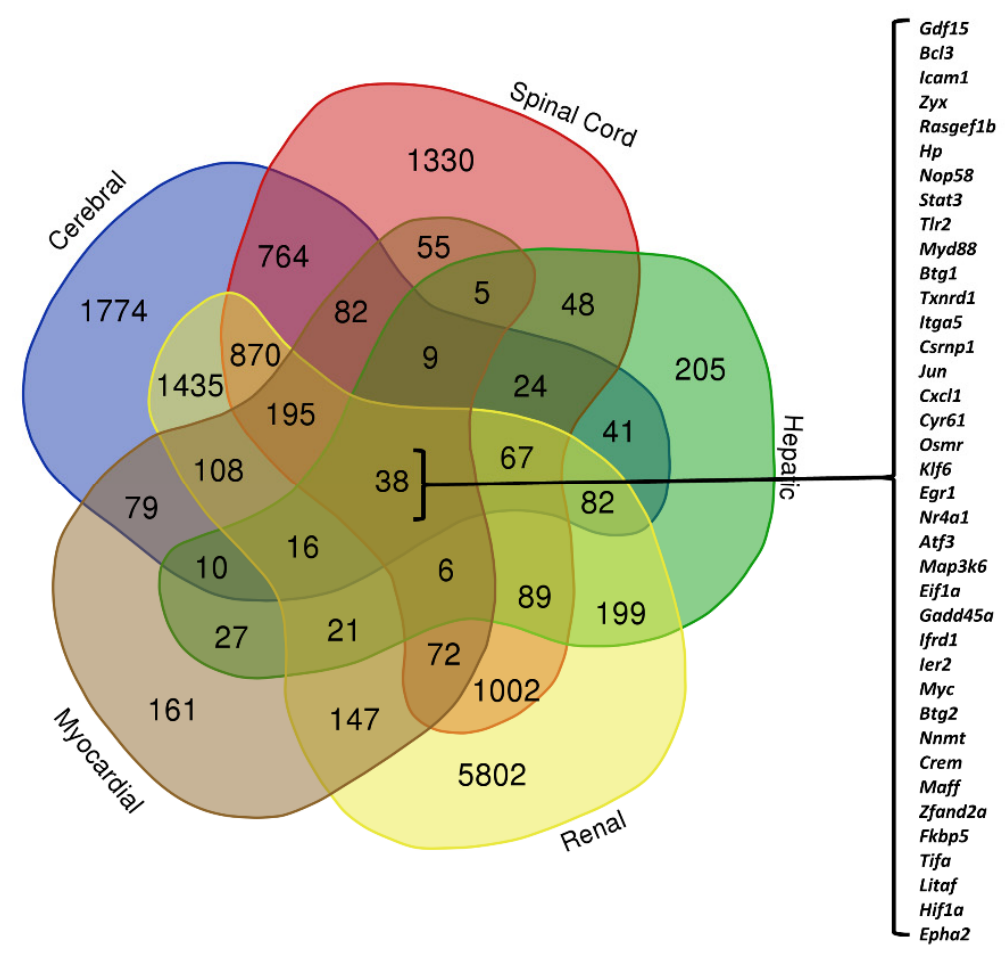

Figure 4. Venn diagram of all included DEGs categorized based on source organ. The values represent the number of DEGs in each division (details of diagram in Table S2).

\section{Discussion}

\subsection{Therapeutic Interventions Act on Genes and Pathways Involved in IRI}

Therapeutic interventions were investigated for their efficacy at attenuating IRI. These have included ischemic pre- and post-conditioning and delivery of stem cells to the affected organs. It is likely that treatment approaches that achieve beneficial effects act on the same genes and pathways implicated in IRI via their counter-regulation. Here, we will compare RNAseq data from therapeutic interventions with the results of our cross-comparison of DEGs and pathways across IRI.

\subsection{Ischemic Postconditioning}

Zhang et al. (2019) used ischemic postconditioning (IPO) of the liver in a wildtype mouse model, whereby, following $1 \mathrm{~h}$ of ischemia, the porta hepatis was re-perfused for three $5 \mathrm{~s}$ cycles prior to full reperfusion for four hours [18]. The extent of injury was then compared between animals that received IPO and those that only underwent IRI. Among those we found to be implicated across IRI conditions, the authors found Cyr61 to be downregulated by IPO. Cyr61 belongs to the $\mathrm{CCN}$ protein family and regulates cell adhesion, proliferation, and apoptosis [36,37]. Additionally, they observed significant upregulation of Atf3 in IPO, the product of which is a member of the ATF/cAMP-responsive element binding protein transcription factor family and has been found to repress macrophage pro-inflammatory gene expression in IRI $[38,39]$. Several pathways the authors found to be differentially regulated in liver IPO were similar to those we found to be highly implicated across IRI studies. IPO significantly downregulated the MAPK and IL-17 signaling pathways, which modulate cell proliferation and apoptosis, and inflammatory cytokines and apoptosis, respectively, suggesting their role as potential protective mechanisms in IRI [18].

\subsection{Caloric and Hypoxic Preconditioning}

Additional conditioning methods, including caloric restriction (CR) and hypoxic preconditioning (HP), were investigated for their efficacy in attenuating renal IRI in wildtype mice [20]. Animals were subjected to either a 30\% reduction in food consumption per 
week or three days of hypoxic exposure for 2,4 , and $8 \mathrm{~h}$ sequentially, prior to undergoing 40 min clamping of the left renal pedicle followed by 4,24 , or $72 \mathrm{~h}$ of reperfusion. CR was found to downregulate apoptotic regulator genes Bik and Dynll1 that were inversely upregulated in IRI. In non-preconditioned animals, IRI was found to upregulate similar pro-inflammatory pathways to those enriched in our KEGG analysis, including NF- $\mathrm{kB}$, TNF, and IL-17. Both HP and CR were found to regulate several genes overlapping with IRI in an inverse manner, providing significant protective effects [20].

\subsection{Stem Cell Therapy Approaches}

Stem cell treatment was also employed to aid in reparative processes and supplement depleted cell populations in order to encourage functional recovery. Using a rodent middle cerebral artery occlusion (MCAO) model of stroke, pluripotent hematopoietic stem/progenitor cells (HSPC) were delivered $24 \mathrm{~h}$ post-reperfusion and were successful in reducing infarct volume and improving neurologic score [29]. HSPC treatment was found to upregulate IL-10 expression, suggesting anti-inflammatory protection by attenuating immune cell recruitment. This coincides with our findings of upregulation of pro-inflammatory IL-10 signaling in IRI. RNA sequencing also identified significantly increased metallothionein (MT-1) in transplanted cells, a cysteine-rich, free radical bindingprotein that is protective in oxidative stress [40]. Further, multiple genes involved in the pro-inflammatory DENN/ERK/MAPK pathway were found to be significantly upregulated in IRI, adding to the DEGs we found to be highly implicated in the MAPK pathway [29].

Culturing methods were compared to determine the efficacy of spheroid 3D cultures, relative to 2D, for facilitating engraftment following transplant. Multipotent human umbilical cord-derived mesenchymal stem cells (UC-MSC) were employed as a protective treatment in a rat model of hepatic IRI, using 2D and 3D cultured cells, as well as untreated controls, in order to determine an optimal treatment approach [30]. Both treatment conditions were found to reduce mRNA expression of pro-inflammatory TNF, and upregulate the expression of potentially protective IL-6 relative to the untreated controls, recapitulating the protective effects of targeting DEGs most highly implicated in IRI. Three-dimensionalcultured MSCs were found to be more effective at attenuating inflammation by acting on the TNF signaling pathway, as illustrated by their upregulation of TNF- $\alpha$-induced protein 8 , a potent inhibitor of apoptosis [41], and TNF- $\alpha$-stimulated gene- 6 , a modulator of macrophage polarization [42]. Further, 3D cultured MSC were found to differentially regulate trophic factors, such as vascular endothelial growth factor, hepatocyte growth factor, and basic fibroblast growth factor, potentially affecting their subsequent engraftment and suggesting a therapeutic target to improve stem cell treatment efficacy [30].

\section{Conclusions}

In summary, IRI inflicts extensive damage by inhibiting vascular circulation, thereby disrupting metabolic and ionic regulation. This is then followed by the restoration of blood flow across oxygen-depleted areas, often overwhelming membrane flux and causing the formation of free radicals. This results in the initiation of several inflammatory kinase pathways as cells respond to ROS-induced damage and attempt to reinstate homeostasis. Several pathways are canonically dysregulated across IRI modalities, with immune signaling and cell death regulating pathways being the primary targets. Those modulating metabolic processes, ionic exchange, and developmental pathways are also highly implicated, as illustrated by functional clusters of genes displaying significant differential expression in ischemia-reperfusion injury models.

Our analysis of transcriptomic changes in IRI across organs was limited by a lack of studies providing comprehensive data on differential gene expression in IRI. Understanding the roles of these genes and pathways at large is a critical step in advancing therapeutic strategies, and the advent of RNA sequencing provides unprecedented insight into these dynamics. In this study, we analyzed differential gene expression and pathway regulation 
across existing studies to provide a broad overview of genes canonically implicated in IRI. Future research may apply these methods to create a more detailed, comprehensive list of transcriptomic changes in response to ischemia-reperfusion. This information may be used to guide research and optimize therapeutic approaches going forward.

Supplementary Materials: The following are available online at https://www.mdpi.com/article/10 .3390 / cells10071838/s1, Table S1: The full list of DEGs reported in each publication, Table S2: The detailed results of Venn diagram, Table S3: The comparison analysis of all DEGs reported in the analysed papers and number of time they were repeated, Table S4: The full list of gene's that were consistently reported to get upregulated in IRI (at least 4 time reported by papers), Table S5: The full list of gene's that were consistently reported to get downregulated in IRI (at least 4 time reported by papers), Table S6: The list of enriched GO terms in Biological processes domain for Upregulated genes, Table S7: The list of enriched GO terms in Cellular Component domain for Upregulated genes, Table S8: The list of enriched GO terms in Molecular Function domain for Upregulated genes, Table S9: The list of enriched GO terms in Biological processes domain for Downregulated genes, Table S10: The list of enriched GO terms in Cellular Component domain for Downregulated genes, Table S11: The list of enriched GO terms in Molecular Function domain for Downregulated genes, Table S12: The list of enriched KEGG pathways for Upregulated genes, Table S13: The list of enriched KEGG pathways for Downregulated genes.

Author Contributions: Conception, data collection, writing-original draft preparation, editing, M.M.; data collection, writing-original draft preparation, editing, S.B.; conception, writing-review and editing, J.H.; conception, editing, review, supervision, financial support, final approval of manuscript, M.G.F. All authors have read and agreed to the published version of the manuscript.

Funding: M.G.F. is supported by the Halbert Chair in Neural Repair and Regeneration and the DeZwirek Family Foundation.

Institutional Review Board Statement: Not applicable.

Informed Consent Statement: Not applicable.

Data Availability Statement: The data presented in this study are available in the supplementary material of this article.

Conflicts of Interest: The authors declare no conflict of interest.

\section{Abbreviations}

\begin{tabular}{|c|c|}
\hline Abbreviation & Explanation \\
\hline ATF & activating transcription factors \\
\hline $\mathrm{BP}$ & biological process \\
\hline CaMK & calcium/calmodulin-dependent protein kinase \\
\hline cAMP & cyclic adenosine monophosphate \\
\hline $\mathrm{CCN}$ & $\begin{array}{l}\text { CYR61 (cysteine-rich angiogenic protein 61), } \\
\text { CTGF (connective tissue growth factor), NOV (nephroblastoma overexpressed) }\end{array}$ \\
\hline $\mathrm{CC}$ & cellular components \\
\hline CR & caloric restriction \\
\hline DEGs & differentially expressed genes \\
\hline DENN & differentially expressed in normal and neoplastic cells \\
\hline ERK & extracellular signal-regulated kinases \\
\hline FDR & false discovery rate \\
\hline GO & Gene Ontology \\
\hline $\mathrm{HP}$ & hypoxic preconditioning \\
\hline HSPC & pluripotent hematopoietic stem/progenitor cells \\
\hline IL-10 & Interleukin-10 \\
\hline IL-12 & Interleukin-12 \\
\hline IL-17 & Interleukin-17 \\
\hline IL-6 & Interleukin-6 \\
\hline IPO & ischemic postconditioning \\
\hline
\end{tabular}




$\begin{array}{ll}\text { IRI } & \text { ischemia reperfusion injury } \\ \text { KEGG } & \text { Kyoto Encyclopedia of Genes and Genomes } \\ \text { MAPK } & \text { mitogen-activated protein kinase } \\ \text { MCAO } & \text { middle cerebral artery occlusion } \\ \text { MF } & \text { molecular function } \\ \text { MSC } & \text { mesenchymal stem cells } \\ \text { MT-1 } & \text { metallothionein } \\ \text { NF-KB } & \text { nuclear factor kappa-light-chain-enhancer of activated B cells } \\ \text { PANTHER } & \text { protein analysis through evolutionary relationships } \\ \text { ROS } & \text { reactive oxidative species } \\ \text { TNF } & \text { tumor necrosis factor alpha } \\ \text { UC-MSC } & \text { umbilical cord-derived mesenchymal stem cells }\end{array}$

\section{References}

1. Kalogeris, T.; Baines, C.; Krenz, M.; Korthuis, R.J. Cell Biology of Ischemia/Reperfusion Injury. Int. Rev. Cell Mol. Biol. 2012, 298, 229-317. [CrossRef]

2. Hausenloy, D.J.; Yellon, D.M. Myocardial ischemia-reperfusion injury: A neglected therapeutic target. J. Clin. Investig. 2013, 123, 92-100. [CrossRef] [PubMed]

3. Nour, M.; Scalzo, F.; Liebeskind, D.S. Ischemia-Reperfusion Injury in Stroke. Interv. Neurol. 2012, 1, 185-199. [CrossRef]

4. Kosieradzki, M.; Rowiński, W. Ischemia/Reperfusion Injury in Kidney Transplantation: Mechanisms and Prevention. In Proceedings of the Transplantation Proceedings; Elsevier BV: Toronto, ON, Canada, 2008; Volume 40, pp. 3279-3288.

5. Kupiec-Weglinski, J.; Busuttil, R. Ischemia and Reperfusion Injury in Liver Transplantation. In Proceedings of the Transplantation Proceedings; Elsevier BV: Toronto, ON, Canada, 2005; Volume 37, pp. 1653-1656.

6. Honda, H.M.; Korge, P.; Weiss, J.N. Mitochondria and Ischemia/Reperfusion Injury. Ann. New York Acad. Sci. 2005, 1047, $248-258$. [CrossRef]

7. Minamino, T.; Komuro, I.; Kitakaze, M.; Minamino, T.; Komuro, I.; Kitakaze, M. Endoplasmic Reticulum Stress as a Therapeutic Target in Cardiovascular Disease. Circ. Res. 2010, 107, 1071-1082. [CrossRef]

8. Lillig, C.H.; Berndt, C.; Holmgren, A. Glutaredoxin systems. Biochim. Biophys. Acta (BBA) Gen. Subj. 2008, $1780,1304-1317$. [CrossRef]

9. Flück, M.; Carson, J.A.; Gordon, S.E.; Ziemiecki, A.; Booth, F.W. Focal adhesion proteins FAK and paxillin increase in hypertrophied skeletal muscle. Am. J. Physiol. Content 1999, 277, C152-C162. [CrossRef]

10. Gundewar, S.; Calvert, J.; Elrod, J.; Lefer, D.J. Cytoprotective effects of N,N,N-trimethylsphingosine during ischemia- reperfusion injury are lost in the setting of obesity and diabetes. Am. J. Physiol. Circ. Physiol. 2007, 293, H2462-H2471. [CrossRef] [PubMed]

11. Degterev, A.; Huang, Z.; Boyce, M.; Li, Y.; Jagtap, P.; Mizushima, N.; Cuny, G.D.; Mitchison, T.J.; Moskowitz, M.; Yuan, J. Chemical inhibitor of nonapoptotic cell death with therapeutic potential for ischemic brain injury. Nat. Chem. Biol. 2005, 1, 112-119. [CrossRef] [PubMed]

12. Murphy, E.; Steenbergen, C. Ion Transport and Energetics During Cell Death and Protection. Physiology 2008, 23, 115-123. [CrossRef]

13. Wu, Y.; Satkunendrarajah, K.; Teng, Y.; Chow, D.S.-L.; Buttigieg, J.; Fehlings, M.G. Delayed Post-Injury Administration of Riluzole Is Neuroprotective in a Preclinical Rodent Model of Cervical Spinal Cord Injury. J. Neurotrauma 2013, 30, 441-452. [CrossRef] [PubMed]

14. Tang, J.; Zhuang, S. Histone acetylation and DNA methylation in ischemia/reperfusion injury. Clin. Sci. 2019, 133, 597-609. [CrossRef] [PubMed]

15. Zhou, Z.; Han, B.; Jin, H.; Chen, A.; Zhu, L. Changes in long non-coding RNA transcriptomic profiles after ischemia-reperfusion injury in rat spinal cord. PeerJ 2020, 8, e8293. [CrossRef]

16. Cai, Y.; Zhang, Y.; Ke, X.; Guo, Y.; Yao, C.; Tang, N.; Pang, P.; Xie, G.; Fang, L.; Zhang, Z.; et al. Transcriptome Sequencing Unravels Potential Biomarkers at Different Stages of Cerebral Ischemic Stroke. Front. Genet. 2019, 10, 814. [CrossRef] [PubMed]

17. Dergunova, L.V.; Filippenkov, I.B.; Stavchansky, V.V.; Denisova, A.E.; Yuzhakov, V.V.; Mozerov, S.A.; Gubsky, L.V.; Limborska, S.A. Genome-wide transcriptome analysis using RNA-Seq reveals a large number of differentially expressed genes in a transient MCAO rat model. BMC Genom. 2018, 19, 655. [CrossRef] [PubMed]

18. Zhang, P.; Ming, Y.; Cheng, K.; Niu, Y.; Ye, Q. Gene Expression Profiling in Ischemic Postconditioning to Alleviate Mouse Liver Ischemia/Reperfusion Injury. Int. J. Med Sci. 2019, 16, 343-354. [CrossRef]

19. Shi, J.; Chen, X.; Li, H.; Wu, Y.; Wang, S.; Shi, W.; Chen, J.; Ni, Y. Neuron-autonomous transcriptome changes upon ischemia/reperfusion injury. Sci. Rep. 2017, 7, 1-11. [CrossRef]

20. Johnsen, M.; Kubacki, T.; Yeroslaviz, A.; Späth, M.R.; Mörsdorf, J.; Göbel, H.; Bohl, K.; Ignarski, M.; Meharg, C.; Habermann, B.; et al. The Integrated RNA Landscape of Renal Preconditioning against Ischemia-Reperfusion Injury. J. Am. Soc. Nephrol. 2020, 31, 716-730. [CrossRef] 
21. Li, X.; Zheng, S.; Tan, W.; Chen, H.; Li, X.; Wu, J.; Luo, T.; Ren, X.; Pyle, G.; Wang, L.; et al. Slit2 Protects Hearts Against Ischemia-Reperfusion Injury by Inhibiting Inflammatory Responses and Maintaining Myofilament Contractile Properties. Front. Physiol. 2020, 11, 228. [CrossRef]

22. Mo, D.; Tian, W.; Zhang, H.-N.; Feng, Y.-D.; Sun, Y.; Quan, W.; Hao, X.-W.; Wang, X.-Y.; Liu, X.-X.; Li, C.; et al. Cardioprotective effects of galectin-3 inhibition against ischemia/reperfusion injury. Eur. J. Pharmacol. 2019, 863, 172701. [CrossRef]

23. Kestner, R.-I.; Mayser, F.; Vutukuri, R.; Hansen, L.; Günther, S.; Brunkhorst, R.; Devraj, K.; Pfeilschifter, W. Gene Expression Dynamics at the Neurovascular Unit During Early Regeneration After Cerebral Ischemia/Reperfusion Injury in Mice. Front. Neurosci. 2020, 14, 280. [CrossRef]

24. Kölling, M.; Genschel, C.; Kaucsar, T.; Hübner, A.; Rong, S.; Schmitt, R.; Sörensen-Zender, I.; Haddad, G.; Kistler, A.; Seeger, H.; et al. Hypoxia-induced long non-coding RNA Malat1 is dispensable for renal ischemia/reperfusion-injury. Sci. Rep. 2018, 8, 3438. [CrossRef]

25. Wang, C.-M.; Pan, Y.-Y.; Liu, M.-H.; Cheng, B.-H.; Bai, B.; Chen, J. RNA-seq expression profiling of rat MCAO model following reperfusion Orexin-A. Oncotarget 2017, 8, 113066-113081. [CrossRef] [PubMed]

26. Park, M.; Kwon, C.H.; Ha, H.K.; Han, M.; Song, S.H. RNA-Seq identifies condition-specific biological signatures of ischemiareperfusion injury in the human kidney. BMC Nephrol. 2020, 21, 1-12. [CrossRef] [PubMed]

27. Giraud, S.; Steichen, C.; Allain, G.; Couturier, P.; Labourdette, D.; Lamarre, S.; Ameteau, V.; Tillet, S.; Hannaert, P.; Thuillier, R.; et al. Dynamic transcriptomic analysis of Ischemic Injury in a Porcine Pre-Clinical Model mimicking Donors Deceased after Circulatory Death. Sci. Rep. 2018, 8, 5986. [CrossRef] [PubMed]

28. Deng, S.; Essandoh, K.; Wang, X.; Li, Y.; Huang, W.; Chen, J.; Peng, J.; Jiang, D.-S.; Mu, X.; Wang, C.; et al. Tsg101 positively regulates P62-Keap1-Nrf2 pathway to protect hearts against oxidative damage. Redox Biol. 2020, 32, 101453. [CrossRef]

29. Smith, H.K.; Omura, S.; Vital, S.A.; Becker, F.; Senchenkova, E.Y.; Kaur, G.; Tsunoda, I.; Peirce, S.M.; Gavins, F.N.E. Metallothionein I as a direct link between therapeutic hematopoietic stem/progenitor cells and cerebral protection in stroke. FASEB J. 2018, 32, 2381-2394. [CrossRef]

30. Sun, Y.; Wang, Y.; Zhou, L.; Zou, Y.; Huang, G.; Gao, G.; Ting, S.; Lei, X.; Ding, X. Spheroid-cultured human umbilical cord-derived mesenchymal stem cells attenuate hepatic ischemia-reperfusion injury in rats. Sci. Rep. 2018, 8, 1-12. [CrossRef]

31. Liu, J.; Kumar, S.; Dolzhenko, E.; Alvarado, G.F.; Guo, J.; Lu, C.; Chen, Y.; Li, M.; Dessing, M.C.; Parvez, R.K.; et al. Molecular characterization of the transition from acute to chronic kidney injury following ischemia/reperfusion. JCI Insight 2017, 2, e94716. [CrossRef] [PubMed]

32. Mi, H.; Muruganujan, A.; Ebert, D.; Huang, X.; Thomas, P.D. PANTHER version 14: More genomes, a new PANTHER GO-slim and improvements in enrichment analysis tools. Nucleic Acids Res. 2019, 47, D419-D426. [CrossRef] [PubMed]

33. R: The R Project for Statistical Computing. Available online: https://www.r-project.org/ (accessed on 2 July 2021).

34. Yu, G.; Wang, L.-G.; Han, Y.; He, Q.-Y. clusterProfiler: An R Package for Comparing Biological Themes Among Gene Clusters. OMICS A J. Integr. Biol. 2012, 16, 284-287. [CrossRef]

35. Zamanian, J.L.; Xu, L.; Foo, L.C.; Nouri, N.; Zhou, L.; Giffard, R.G.; Barres, B.A. Genomic Analysis of Reactive Astrogliosis. J. Neurosci. 2012, 32, 6391-6410. [CrossRef] [PubMed]

36. Grzeszkiewicz, T.M.; Lindner, V.; Chen, N.; Lam, S.C.-T.; Lau, L.F. The Angiogenic Factor Cysteine-Rich 61 (CYR61, CCN1) Supports Vascular Smooth Muscle Cell Adhesion and Stimulates Chemotaxis through Integrin $\alpha 6 \beta 1$ and Cell Surface Heparan Sulfate Proteoglycans. Endocrinology 2002, 143, 1441-1450. [CrossRef]

37. Chen, Y.; Du, X. Functional properties and intracellular signaling of CCN1/Cyr61. J. Cell. Biochem. 2007, 100, 1337-1345. [CrossRef]

38. De Nardo, D.; Labzin, L.; Kono, H.; Seki, R.; Schmidt, S.V.; Beyer, M.; Xu, D.; Zimmer, S.; Lahrmann, C.; Schildberg, F.A.; et al. High-density lipoprotein mediates anti-inflammatory reprogramming of macrophages via the transcriptional regulator ATF3. Nat. Immunol. 2014, 15, 152-160. [CrossRef] [PubMed]

39. Cheng, C.-F.; Lin, H. Acute kidney injury and the potential for ATF3-regulated epigenetic therapy. Toxicol. Mech. Methods 2011, 21, 362-366. [CrossRef] [PubMed]

40. Ruttkay-Nedecky, B.; Nejdl, L.; Gumulec, J.; Zitka, O.; Masarik, M.; Eckschlager, T.; Stiborova, M.; Adam, V.; Kizek, R. The Role of Metallothionein in Oxidative Stress. Int. J. Mol. Sci. 2013, 14, 6044-6066. [CrossRef]

41. Zhang, L.; Liu, R.; Luan, Y.-Y.; Yao, Y.-M. Tumor Necrosis Factor- $\alpha$ Induced Protein 8: Pathophysiology, Clinical Significance, and Regulatory Mechanism. Int. J. Biol. Sci. 2018, 14, 398-405. [CrossRef] [PubMed]

42. Dixon, M.L.; De La Vega, A.; Mills, C.; Andrews-Hanna, J.; Spreng, R.N.; Cole, M.W.; Christoff, K. Heterogeneity within the frontoparietal control network and its relationship to the default and dorsal attention networks. Proc. Natl. Acad. Sci. USA 2018, 115, E1598-E1607. [CrossRef] [PubMed] 